\title{
CORNEAL TOPOGRAPHY IN VERNAL KERATOCONJUNCTIVITIS (VKC)
}

\author{
Atul Gupta1, S. Sravanthi²
}

${ }^{1}$ Associate Professor, Department of Ophthalmology, Government Medical College, Nizamabad, Telangana. 2Senior Resident, Department of Ophthalmology, Government Medical College, Nizamabad, Telangana.

\section{BACKGROUND}

\section{ABSTRACT}

Vernal keratoconjunctivitis (VKC) is a seasonally recurring bilateral inflammation of cornea and conjunctiva found in individuals with atopic background characterised by symptoms like itching, watering, foreign body sensation. Chronic epithelial trauma provoked by eye rubbing due to intense ocular itching has been postulated as important risk factor in the pathogenesis of keratoconus.

Our aim was to detect anterior corneal surface changes like astigmatism and keratoconus by topographic evaluation of cornea.

\section{MATERIALS AND METHODS}

This is a descriptive study carried out on 100 patients of VKC of age below 15 years at Sarojini Devi Eye Hospital between August 2016 and July 2017. Best corrected visual acuity (BCVA), slit lamp biomicroscopy and corneal topography were done in each patient. Parameters like steep K, flat K and astigmatism were analysed. Keratoconus like topography were noted.

\section{RESULTS}

Out of 100 patients, 77 were males and 33 were females. Majority were in the age group of 5 - 10 years. Majority of patients presented with mixed type, i.e. $62 \%$. Out of 100 patients, astigmatism < 1 D which is physiological was seen in $50 \%$ (Males- 37 , Females- 13) of patients, astigmatism 1-2 D was seen in 29\% (Males- 22, Females- 7) of patients, astigmatism $>2 \mathrm{D}$ was seen in 14\% (Males- 11, Females- 4) of patients and Steep K > 47.2 D + astigmatism > 1.5 D i.e. keratoconus like topography was seen in $7 \%$ (Males- 7) of patients.

\section{CONCLUSION}

Incidence of astigmatism and keratoconus in VKC patients is more when compared to general population of same age group.

\section{KEY WORDS}

Vernal Keratoconjunctivitis (VKC), Topography, Astigmatism, Keratoconus.

HOW TO CITE THIS ARTICLE: Gupta A, Sravanthi S. Corneal topography in vernal keratoconjunctivitis (VKC). J. Evolution Med. Dent. Sci. 2018;7(19):2351-2354, DOI: 10.14260/jemds/2018/530

\section{BACKGROUND}

Vernal keratoconjunctivitis (VKC) is also known as spring catarrh. It is a chronic, bilateral, conjunctival inflammatory condition found in individuals predisposed by their atopic background. ${ }^{1}$ It is common in warm, temperate climates during spring and summer season. The onset of disease is generally before age of 10 , it lasts for 2 to 10 years and it usually resolves during late puberty. ${ }^{2}$ Seasonal incidence is $0.1 \%$ to $0.5 \%$ in ocular diseases.

VKC is characterised by symptoms like itching, photophobia, watering, foreign body sensation, ptosis, thick mucus discharge, blepharospasm and signs like confluent papillary hypertrophy on the limbus and tarsal conjunctiva. ${ }^{3}$

Although, the diagnosis of VKC is easy, it remains an unresolved problem due to frequent recurrence. ${ }^{4}$

Vernal keratoconjunctivitis differs from seasonal allergic conjunctivitis and perennial allergic conjunctivitis, because it is a condition mediated by Th2 lymphocytes. However, the

'Financial or Other Competing Interest': None.

Submission 01-03-2018, Peer Review 24-04-2018,

Acceptance 30-04-2018, Published 07-05-2018.

Corresponding Author:

S. Sravanthi,

D/o. S. Rajender Gupta,

H. No. 4-1-77, Achanpally, Bodhan Dist.,

Nizamabad-503185,

Telangana.

E-mail: sravanthisingarapu@gmail.com

DOI: $10.14260 /$ jemds $/ 2018 / 530$

\section{(c) $(1)$}

precise roles of mast cells, eosinophils, fibroblasts and their cytokines in the inflammatory process and the remodelling of conjunctival tissue remain poorly established.5-7

VKC is associated with complications like superficial punctate keratitis with superficial pannus, pseudogerontoxon, shield ulcer, peripheral corneal stromal degeneration leading to astigmatic type of refractive error, keratoconus which leads to visual impairment.

Our aim was to detect corneal surface changes like astigmatism and keratoconus in VKC subjects by quantitative analysis of topographic map.

\section{MATERIALS AND METHODS}

This is a descriptive study carried out on 100 patients of Vernal keratoconjunctivitis (200 eyes) attending the outpatient department of Paediatrics and Squint, Sarojini Devi Eye Hospital between August 2016 and July 2017. Patients were in the age group of 4 - 15 years. Both genders were selected for the study. The study was approved by the ethical review board of the institute and an informed consent was obtained from all the participating patients.

The patients or their parents were interviewed as to details of history including age of onset of itching and visual difficulties, estimated frequency of abnormal eye rubbing, previous treatment and presence of allergic disease.

Each subject underwent a detailed ocular examination including recording of best corrected visual acuity (BCVA), slit lamp biomicroscopy and corneal topography. 
The diagnosis of VKC was made on the basis of the typical clinical history of severe itching with characteristic signs including giant papillae on the upper palpebral conjunctiva, limbal infiltrates and eosinophilic concretions (HornerTrantas dots). Additional corneal signs including pseudogerontoxon, shield ulcer and punctate epithelial keratitis were noted. Then, VKC was categorised as either palpebral, limbal or mixed.

Slit-lamp biomicroscopic findings like Vogt's striae, Fleischer's ring, stromal thinning and stromal scarring and findings consistent with diagnosis of keratoconus were recorded for each subject.

Topography was done in each patient using placid discbased Atlas Model 9000 Corneal Topographer. Four keratographic images ensuring proper fixation were taken. One keratograph of each eye was chosen for analysis based on the criteria: the least eyelid shadow to allow proper centration, proper focusing with thin regular continuous rings that covered the cornea from limbus to limbus and absence of any dry spots (discontinuous rings) or excess pooling of tears along the inferior lid margin.

The parameters analysed were Steep K, Flat K and astigmatism. Keratoconus like topography include central corneal power value greater than 47.2 D, Sim-K astigmatism greater than $1.5 \mathrm{D}$, difference in central corneal power between two eyes more than 2D.

Based on these, patients were categorised into astigmatism < 1D, astigmatism 1-2D, astigmatism > 2D, keratoconus like topography (steep $\mathrm{K}>47.2 \mathrm{D}+$ astigmatism $>1.5 \mathrm{D})$.

\section{RESULTS}

A total of 100 patients of VKC were studied. The parameters analysed were slit lamp biomicroscopy findings, topographic parameters- Steep K, Flat K and astigmatism.

\section{Age Distribution}

Demographic profile of the patients is shown in Table 1. In our study population, majority of patients were in the age group of 5 - 10 years, i.e. $48 \%$.

Out of 100 , majority were males $(77 \%)$ and $33 \%$ were females.

\begin{tabular}{|c|c|c|c|}
\hline Age Group & Males & Females & Total (\%) \\
\hline $0-5$ & 10 & 6 & $16 \%$ \\
\hline $5-10$ & 37 & 11 & $48 \%$ \\
\hline $10-15$ & 30 & 6 & $36 \%$ \\
\hline \multicolumn{4}{|c|}{ Table 1 } \\
\hline
\end{tabular}

\section{Duration of Symptoms}

\begin{tabular}{|c|c|}
\hline Topography & Mean Duration of Symptoms \\
\hline Astigmatism < 1D & 6.3 months \\
\hline Astigmatism 1-2D & 9.3 months \\
\hline Astigmatism > 2D & 15.3 months \\
\hline $\begin{array}{c}\text { Steep K > 47.2D + } \\
\text { Astigmatism > 1.5D }\end{array}$ & 5.4 years (64 months) \\
\hline \multicolumn{2}{|c|}{ Table 2 } \\
\hline
\end{tabular}

\section{Analysis of Presenting Symptoms}

The most common presenting symptoms observed were itching, watering, redness and FB sensation being seen in $92 \%, 87 \%, 86 \%$ and $68 \%$ of the patients.

\section{Clinical Form of VKC}

Majority of patients were presented with mixed type, i.e. $62 \%$, palpebral in $29 \%$ and limbal type in $9 \%$ of patients.

\section{Topography}

Out of 100 patients, astigmatism < 1D which is physiological was seen in 50\% (Males- 37, Females- 13) of patients, astigmatism 1 - 2D was seen in 29\% (Males- 22, Females- 7) of patients, astigmatism $>2 \mathrm{D}$ was seen in $14 \%$ (Males- 11 , Females- 4) of patients and Steep K $>47.2 \mathrm{D}+$ astigmatism $>1.5 \mathrm{D}$ i.e. keratoconus like topography was seen in $7 \%$ (Males- 7) of patients.

\begin{tabular}{|c|c|c|c|}
\hline Topography & Males & Females & Total (\%) \\
\hline Astigmatism < 1D & 37 & 13 & 50 \\
\hline Astigmatism 1 - 2D & 22 & 6 & 28 \\
\hline Astigmatism > 2D & 11 & 3 & 14 \\
\hline $\begin{array}{c}\text { Steep K > 47.2D+ } \\
\text { Astigmatism > 1.5D }\end{array}$ & 7 & 1 & 8 \\
\hline \multicolumn{3}{|c|}{ Table 3 } \\
\hline
\end{tabular}

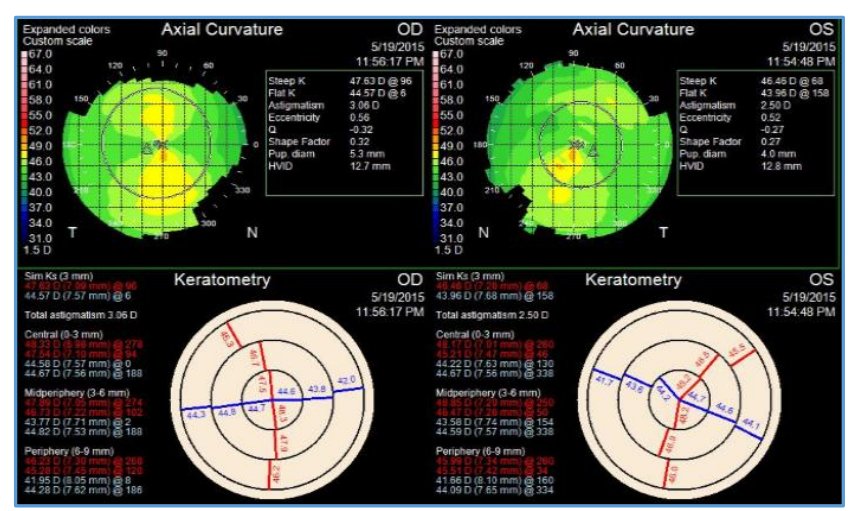

Topography showing Astigmatism

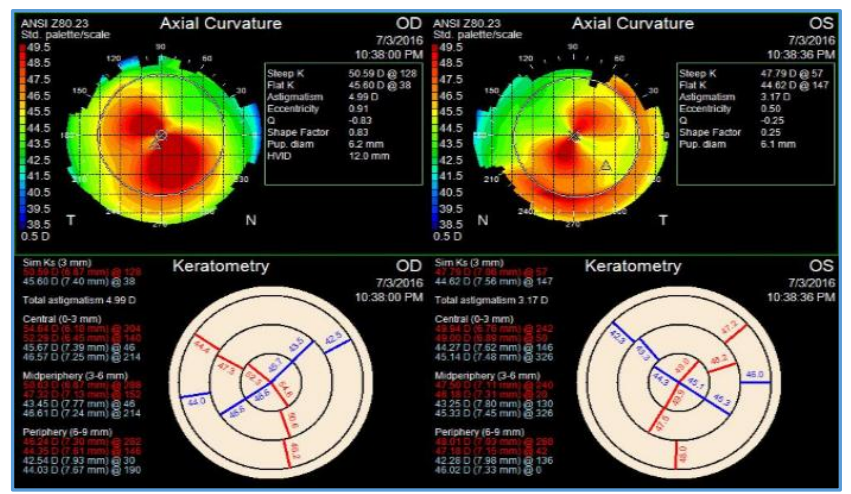

Topography showing Asymmetrical Bow Tie Pattern with Skewing and Steepening

\section{DISCUSSION}

Vernal keratoconjunctivitis is known to be one of the most severe forms of ocular allergy with potential to cause corneal damage and permanent visual loss.

Corneal involvement occurs in 50\% patients of VKC. They are superficial punctate keratitis with superficial pannus, pseudogerontoxon, shield ulcer, peripheral corneal stromal degeneration leading to astigmatic type of refractive error, keratoconus.

Keratoconus and Eye Rubbing

Diverse mechanisms have been associated with the 
development of keratoconus. These include increased inflammatory mediators and loss of keratocytes, abnormal enzyme function and loss of collagen, anomalous keratocyte function and altered fibrillogenesis, altered proteoglycan production, reduced tensile strength, collagen slippage in cone, and cone formation by biomechanically coupled curvature transfer.

Rubbing has been shown to degranulate palpebral conjunctival mast cells in animal studies and histamine release may perpetuate itch cycles, and corresponding additional rubbing episodes. Prostaglandins may be released during episodes acting directly to induce itching in conjunctiva causing a vicious cycle. Allergy associated substances derived from eosinophils were detected in tears and have been shown to have cytotoxic effects on corneal epithelium.

Mechanical trauma leads to biomechanical and biochemical alterations. Epithelial microtrauma leads to cytokine release, especially an increased Interleukin-1 (IL-1) from the epithelium. IL-1 is postulated to be a modulator of epithelial stromal interactions with a role in regulation of corneal cell proliferation, differentiation and death. It induces keratocytes death in vitro and negative keratocytes chemotaxis.

One hypothesis states that the imbalance between keratocyte apoptosis and proliferation may be the pathophysiologic mechanism of KC. Corneal fibroblast in KC eyes have much more IL-1 receptors than healthy eyes, increased expression of IL-1 receptors sensitises the keratocytes causing a loss of keratocytes through apoptosis and in stromal mass resulting in stromal thinning.

The purpose of this study was to evaluate the incidence of astigmatism, incidence of keratoconus in VKC patients by quantitative analysis of topographic maps, to detect clinical characteristics of VKC associated keratoconus.

In our study out of 100 patients, $77 \%$ were males, $23 \%$ were females, male-to-female ratio of being 3.3 which correlates with the study "Ocular complications of severe Vernal Keratoconjunctivitis in Yemen"8 conducted by Saleh et al in 430 patients of VKC showed that majority of VKC patients were males with male: female ratio of 3.1: 1 .

Leonardi ${ }^{9}$ and co-workers in two separate observations including a multicentric study from Italy found $\mathrm{M}$ : $\mathrm{F}$ ratio between 3.3 and 3.5. By and large, all other series have reported M: F ratio between 4: 1 and 2: 1 . Mean age at presentation was 9 years.

The most common presenting symptoms observed were itching, watering, redness and FB sensation being seen in $92 \%, 87 \%, 86 \%$ and $68 \%$ of the patients. Symptoms were seasonal in $72 \%$ patients and throughout the year in $28 \%$ patients.

Of the subjects, $68 \%$ of patients were noted to rub their eyes frequently (i.e. 10 times daily), 23\% rubbed their eyes occasionally (i.e. 5 - 10 times daily) and 9\% rarely (i.e. 5 times daily).

Allergic history was noted in $7 \%$ patients. Out of $7 \%, 3 \%$ had history of skin allergy, $2 \%$ had history of respiratory tract symptoms like asthma, allergic rhinitis and $2 \%$ had history of allergy for specific food. Family history was noted in $5 \%$ patients.

The majority $66 \%$ of patients had a mixed form of disease with involvement of both limbal and palpebral areas. Isolated palpebral form of VKC was present in $23 \%$ patients and isolated limbal form was seen in $11 \%$ which correlates with the study of demographic and clinical profile of VKC in India conducted by Saboo et al ${ }^{10}$ in 468 patients of VKC, which states that majority $72 \%$ of patients have mixed form of disease.

Out of 100 patients $28 \%$ of patients had complications of keratopathy such as $9 \%$ had punctuate keratitis, $15 \%$ had pseudogerontoxon, $4 \%$ had shield ulcer which correlates with Totan et al ${ }^{11}$ study conducted on 82 patients of VKC which showed 31\% keratopathy related complications.

Slit lamp biomicroscopy findings like Vogt's striae and stromal thinning were seen in $6 \%$ of patients.

Based on topography, astigmatism 1 - 2D was seen in $28 \%$ of patients (22 males, 6 females), > 2D astigmatism was seen in $14 \%$ of patients ( 11 males, 3 females) which is significantly higher when compared to general population.

Astigmatism in rural population in India according to Dandona et al ${ }^{12}$ is $2.8 \%$ and astigmatism in urban population according to Murthy et $\mathrm{al}^{13}$ is $5.4 \%$. Our study includes a mixed population (rural and urban) of VKC patients and shows astigmatism of 1 - 2D in $28 \%$ of patients, > 2D in $14 \%$ of patients which is higher when compared to both studies conducted on general population.

The study of Vernal keratoconjunctivitis in school children in Rwanda conducted by De Smedt SK et al ${ }^{14}$ states that children with VKC were $>6$ times more likely to have corneal astigmatism $>2 \mathrm{D}$ in worse eye.

Mean duration of symptoms in group of patients with astigmatism of $1-2 \mathrm{D}$ is 9.3 months and in group of patients with astigmatism $>2 \mathrm{D}$ is 15.3 months, which indicates that longer the duration of symptoms more the chances of corneal changes.

Keratoconus based on slit lamp biomicroscopy along with supportive topographic findings was seen in $8 \%$ of patients.

Keratoconus like topography include central corneal power value greater than 47.2 D, Sim-K astigmatism greater than $1.5 \mathrm{D}$, difference in central corneal power between two eyes more than 2D. We could not calculate Inferior-Superior (I-S) asymmetry as our topography machine does not give point to point keratometry value and could not calculate radial skewing of axis as our machine does not provide necessary parameters required for that.

Out of $8 \%, 7 \%$ of patients were males and $1 \%$ of patients were females. The male predominance in the study in combined VKC and keratoconus cases reflects the high prevalence of VKC in males, which is in agreement with the previous studies,15,9 but not with marked female preponderance generally reported in subjects with keratoconus ${ }^{16,17}$ without VKC.

Mean age of presentation was 12.8 years (range 11 - 15 years). Mean duration of symptoms was 5.4 years.

Based on steep $\mathrm{K}$ value, keratoconus like topography is divided into mild - < 48D, moderate - 48D to 54D, severe $>54 \mathrm{D}$.

Out of $8 \%, 2 \%$ had mild keratoconus, $3 \%$ had moderate keratoconus, $3 \%$ had severe keratoconus.

Keratoconus is a bilateral asymmetrical disease and there is a strong association between VKC, atopy and keratoconus. In a series of 530 cases of VKC examined with slit-lamp biomicroscope and keratoscopy, Khan et al reported a $7 \%$ incidence of keratoconus. Totan et al in a videokeratographic 
study conducted on 82 patients of VKC reported a $26.8 \%$ incidence of keratoconus in VKC. In our study, the incidence of keratoconus detected by topography in VKC was $8 \%$ which correlates with Khan et al study, but does not correlate with Totan et al study, which shows higher incidence. The lower incidence rate in our study may have resulted from the younger age of VKC patients, i.e. below 15 years were included in the study, whereas in other studies the mean age was 15 (range, 8 -22) years, as the duration of disease plays an important role in VKC induced keratoconus.

Long-term disease with a chronic traumatic factor on the corneal epithelium could be related to keratoconus, because as Kim et al pointed out - persistent and chronic corneal trauma on the corneal epithelium (in this particular situation, itching or chronic trauma provoked by giant papillae) induces a "silent" and chronic inflammatory process, leading to progressive loss of stromal mass and consequently to less biomechanical resistance, and thus to anterior corneal steepening, decreasing the optical competence of the anterior corneal surface. 4

\section{Limitations of the Study}

Children with VKC of age below 15 years were included in the study, as it was conducted under Paediatric Ophthalmology department.

\section{CONCLUSION}

- Incidence of Vernal keratoconjunctivitis is more in males than females, hence the topographic changes in cornea are more in males than females.

- Incidence of astigmatism and keratoconus in VKC patients is more when compared to general population of same age group.

- Longer duration of disease is associated with more changes in curvature of cornea.

\section{REFERENCES}

[1] Duke-Elder S, Leigh AG. Disease of the outer eye. Cornea and sclera. Part 2. In: Duke-Elder S. edr. The system of Ophthalmology. Vol. 8. St. Louis: CV Mosby Co, 1965: p. 573.

[2] Bonini S, Coassin M, Aronni S, et al. Vernal keratoconjunctivitis. Eye (Lond) 2004;18(4):345-51.

[3] Müller GG, José NK, De Castro RS. Topical tacrolimus $0.03 \%$ as sole therapy in vernal keratoconjunctivitis: a randomized double-masked study. Eye \& Contact Lens 2014;40(2):79-83.
[4] Allansmith MR. Vernal conjunctivitis. Chapter - 9. In: Duane T. edr. Ophthalmology. Vol. 4. 1982.

[5] Kumagai N, Fukuda K, Fujitsu Y, et al. Role of structural cells of the cornea and conjunctiva in the pathogenesis of vernal keratoconjunctivitis. Prog Retin Eye Res 2006;25(2):165-87.

[6] Bonini S, Lambiase A, Sgrulletta R, et al. Allergic chronic inflammation of the ocular surface in vernal keratoconjunctivitis. Curr Opin Allergy Clin Immunol 2003;3(5):381-7.

[7] Abelson MB, Schaefer K. Conjunctivitis of allergic origin: Immunologic mechanisms and current approaches to therapy. Surv Ophthalmol 1993;38 (Suppl 2):115-32.

[8] Al-Akily SA, Bamashmus MA. Ocular complications of severe vernal keratoconjunctivitis (VKC) in Yemen. Saudi Journal of Ophthalmology 2011;25(3):291-4.

[9] Leonardi A, Busca F, Motterle L, et al. Case series of 406 vernal keratoconjunctivitis patients: a demographic and epidemiological study. Acta Ophthalmol Scand 2006;84(3):406-10.

[10] Saboo US, Jain M, Reddy JC, et al. Demographic and clinical profile of vernal keratoconjunctivitis at a tertiary eye care center in India. Indian J Ophthalmol 2013;61(9):486-9.

[11] Totan Y, Hepsen IF, Cekic O, et al. Incidence of keratoconus in subjects with vernal keratoconjunctivitis: a videokeratographic study. Ophthalmology 2001;108(4):824-7.

[12] Dandona R, Dandona L, Srinivas M, et al. Refractive error in children in a rural population in India. Invest Ophthal Vis Sci 2002;43(3):615-22.

[13] Murthy GVS, Gupta SK, Ellwein LB, et al. Refractive error in children in an urban population in New Delhi. Invest Ophthalmol Vis Sci 2002;43(3):623-31.

[14] De Smedt SK, Nkurikiye J, Fonteyne YS, et al. Vernal keratoconjunctivitis in school children in Rwanda: clinical presentation, impact on school attendance, and access to medical care. Ophthalmology 2012;119(9):1766-72.

[15] Khan MD, Kundi N, Saeed N, et al. Incidence of keratoconus in spring catarrh. $\mathrm{Br} \mathrm{J}$ Ophthalmol 1988;72(1):41-3.

[16] Cameron JA, Al-Rajhi AA, Badr IA. Corneal ectasia in vernal keratoconjunctivitis. Ophthalmology 1989;96(11):1615-23.

[17] Sorsby A. Modern Ophthalmology. Chap - 1. Vol. 3. 2nd edn. London: Butterworths 1972: p. 244. 